\title{
Functional Approaches to Fibromyalgia Syndrome
}

\author{
Seihwan Choi ${ }^{凶}$ \\ St. Mary's Neurosurgical Clinic, Seoul, Korea
}

In the case of acute pain, its cause tends to be precise, so finding it out and taking some rest along with non-steroidal anti-inflammatory drugs (NSAIDs) are enough to sort it out. However with chronic pains, not only that its cause is obscure but also the grade of pain is sensitive to pathological, environmental and psychological changes, bringing various systemic symptoms. Reactions to drugs are more responsive to antianxiety drugs or antidepressants rather than to NSAIDs. This styles of prescription could not be fundamental. Besides, long-term usage of drugs such as NSAIDs are reported to increase the incidences of gastritis, gastrointestinal hemorrhage and even mortalities by recurrence of myocardial infarction. According to the recent studies of fibromyalgia syndrome, one of the common examples of chronic pain, systemic symptoms are known to occur under excessive psychological and oxidative stress. In evaluating the causes of chronic pain, executing the analysis of functional medicine such as comprehensive metabolic profiles, salivary free cortisol, etc. to examine the grade and status of stress response could be of great help. It is highly considerable to try treatments to normalize the abnormalities of the test results, to educate patients' life style, and to try out mind-body therapy in order to treat the underlying causes of chronic pains, so to speak fibromyalgia syndrome.

Key Words: Fibromyalgia syndrome; Chronic pain; Functional medicine; Nutritional therapy; Oxidative stress; Salivary hormone; Mind-body therapy.

凶Corresponding author: Seihwan Choi, Seoul St. Mary's Neurosurgical Clinic, 206 Hakdong-ro, Gangnamgu, Seoul 06052, Korea. Tel: 82-2-3481-8275, Fax: 82-2-3481-8279, E-mail: spinexp@gmail.com

The very evidence that all living organisms are alive is that they can produce a response to external and internal stimuli. These stimuli makes discomfort and when it gets intensified to the degree where it disturbs daily life, we call them 'pain.' In terms of the duration of pain, the pain is sorted into acute and chronic pain, and although these two look similar, they assume very different aspects. Generally, the duration of pains within three months is classified as acute pains and those above three months would be chronic pains. Once again, chronic pains are divided into chronic malignant pain and chronic non-malignant pain. Usually, the chronic pains accompany various symptoms such as decreased appetite, indigestion, failure to thrive, autonomic dysreflexia, depression, insomnia, constipation, etc., so it is often called chronic pain syndrome (CPS).

Most acute pains are easy to find their cause of pain, and the majority of them are known to be controlled when treated with remedy of cause along with enough rest, adequate analgesics and anti-inflammatory drugs. However, different from acute pains, the chronic pains can linger after being treated with remedy of cause and they are known to react better with antianxiety or an- tidepressant drugs. While treating these pains, that seem to not be easily solved to the bottom, in case of the US of 2008, the death toll by drug abuse unfortunately surpassed that of traffic accidents (Fig. 1) $)^{4}$.

According to Gislason" ${ }^{9)}$ the risk of death is said to be continuing on patients with myocardial infarction who are treated with common NSAIDs, after they are traced from one-week to fiveyear term. This risk of death particularly arose when they were prescribed with COX-2 inhibitors in order to reduce complications such as gastritis or gastric ulcer. Among these inhibitors, diclofenac is even more perilous, so it was recommended by Health Canada to limit the dosage of medicines containing diclofenac from $150 \mathrm{mg}$ to $100 \mathrm{mg}^{8-10)}$.

Many diverse influences are responsible for the current state of the public health (Fig. 2) ${ }^{20,22,30)}$. However, it is not enough to demonstrate that the majority of today's chronic diseases could be prevented or ameliorated by changes in lifestyle ${ }^{5)}$. We must also ask what contributes to such unhealthy lifestyles. It is critical that we understand how great a proportion of environment and lifestyle is influenced by conditions beyond the control of 


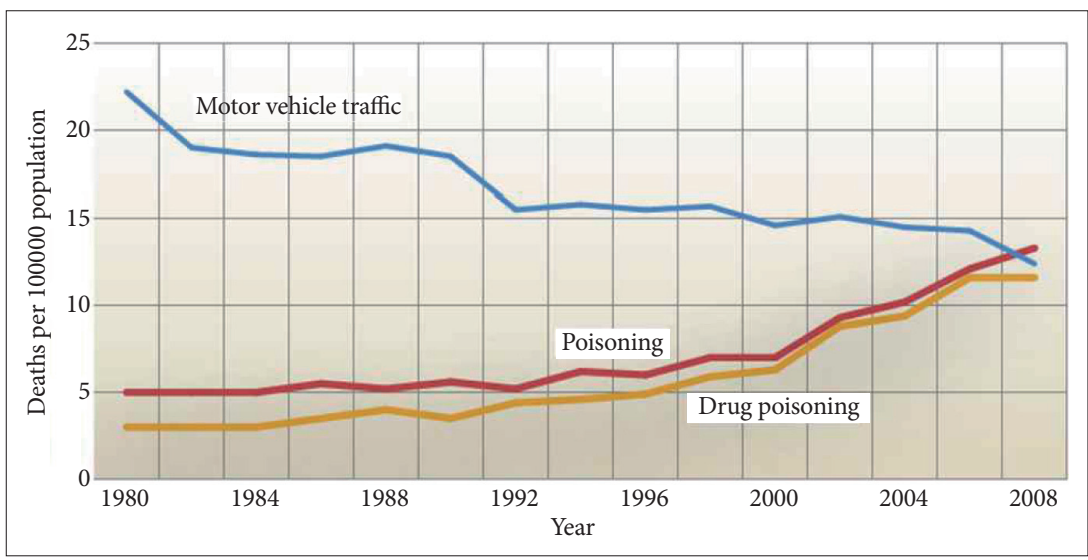

Fig. 1. Motor vehicle traffic, poisoning, and drug poisoning death rates : United States, 1980-2008. NOTE : In 1999, the International Classification of Diseases, Tenth Revision (ICD-10) replaced the previous revision of the ICD (ICD-9). This resulted in approximately $5 \%$ fewer deaths being classified as motor-vehicle traffic-related deaths and $2 \%$ more deaths being classified as poisoning-related deaths. Therefore, death rates for 1998 and earlier are not directly comparable with those computed after 1998. SOURCE : CDC/NCHS, National Vital Statistics System.

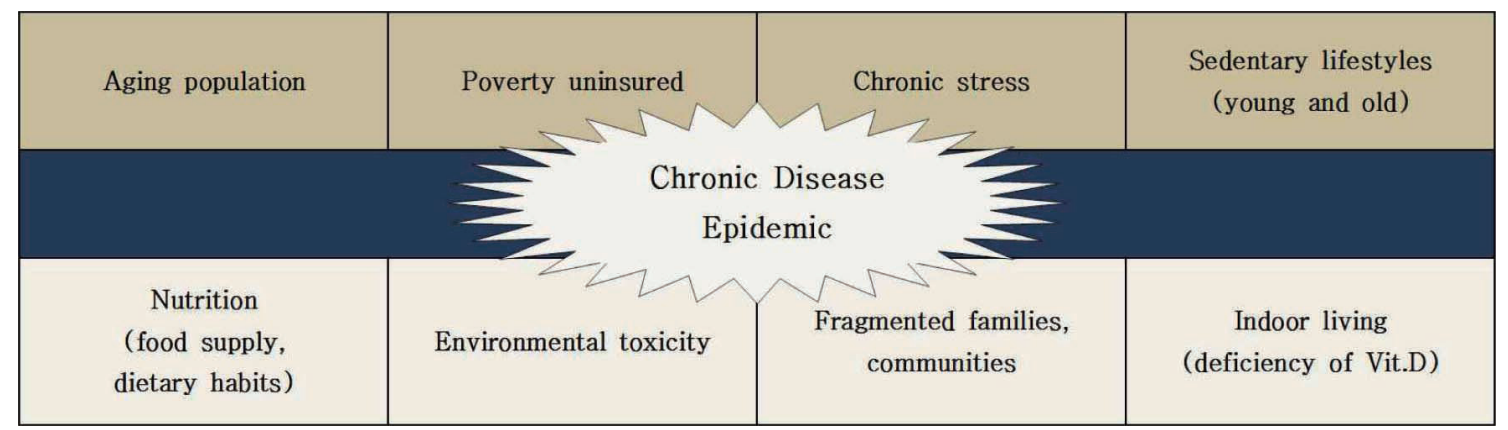

Fig. 2. Major Influences Contributing to the Epidemic of Chronic Disease ${ }^{32)}$ (21st century medicine, A New Model for Medical Education and Practice, xii 2009 IFM, David S. Jones, MD).

individual patients-not only the genetic vulnerability one is born with, but increases in environmental toxicity, the homogenization and denaturing of the food supply, the influence of sedentary technology on jobs, education, and entertainment, the powerlessness and despair of poverty, the debility produced by chronic stress, and the fragmentation of family and community life that leads to isolation and a lessened sense of purpose and meaning. These are all complex problems that took many decades to appear and that will require a long-term national effort and effective leadership in public policy to be solved. We recognize and emphasize that not only must we change healthcare and medical education, but over the next decades we must also change the practices and priorities of our political, social, and economic structures to achieve fundamental change in the public's health. Therefore, the medical education should prepare students well for the clinical problems they will face in their future practice. However, that is not happening for the most prevalent problem in health care today : chronic disease. The reason is that first, chronic disease replaced acute disease as the dominant health problem. Chronic disease is now the principal cause of disability and use of health services, and consumes $78 \%$ of health expenditures in USA. Also chronic disease dramatically transformed the role of the patient ${ }^{11)}$.

\section{THE PHARMACEUTICAL AND ACUTE-CARE MODELS}

The acute-care model is characterized by rapid differential diagnosis aimed at prescribing a drug (or procedure) that will relieve the patient's presenting symptoms and avert the immediate threat ${ }^{14)}$. It minimizes the involvement of the patient, who functions as a mostly passive recipient of the procedure or prescription $^{6}$. It is not a model that reimburses the practitioner for looking into why the patient became ill, or whether she/he will be back many times for ramifications of the same underlying problem $^{11)}$. Instead, it prioritizes quick solutions to the most pressing problems. It is, of course, absolutely essential in emergency and hospital-based care, but difficulties arise when this model is applied to ongoing, community-based care, a process that accelerated under the managed-care movement (which turned out to be far more about managing costs than managing care) and the direct-to-patient advertising of drugs. In short, the acute-care model's treatment mainly depends on pharmaceutical and surgical approaches.

\section{WHAT IS FUNCTIONAL MEDICINE?}

Now, however, in the 21st century, we are fully aware that complex, lifelong interactions between our genes and environ- 
mental degradation ${ }^{13)}$, unhealthy diets ${ }^{23)}$ (fueled by changes in both eating habits and food supply), stress ${ }^{7,15,16)}$, sedentary lives ${ }^{17}$, and social fragmentation of families and communities have surged to the forefront as interwoven causes of chronic disease that are not amenable to treatment with an acute-care model. With an aging population, these effects are present through many more years of life and thus become impressive cost drivers. The system must expand to address these interconnected trends. Broad-based pattern recognition and communications skills will be needed to prevent, treat, and reverse the declining function associated with these pervasive influences. We must transform our system of health care through new models for medical education, acute and chronic disease management, research, health insurance, and fiscal responsibility. In other words, the chronic care model is to make a new paradigm different from conventional treatments by designing a new doctorpatient role model in which patients' lifestyles are modified and they would be educated and empowered, all for the sake of disease prevention.

As cutting-edge technologies developed in the 21st century, information on vital phenomena handled only in laboratories has become more easily available on a clinical level. Using these informations, we have come with more cases where the fundamental causes of human diseases are analysed to make possible the remedy of cause and also its prevention. The IFM (Institute for Functional Medicine; www.functionalmedicine.org) stated that seven clinical imbalances become the cause of diseases, and these factors are interwoven to each other making mutual influences (Fig. 3).

Functional medicine is a science-based personalized health- care approach that assesses and treats underlying causes of illness through individually tailored therapies to restore health and improve function.

\section{DEFINITION OF FMS}

Fibromyalgia (FM or FMS) is characterized by chronic widespread pain and allodynia (a heightened and painful response to pressure). Fibromyalgia symptoms are not restricted to pain, leading to the use of the alternative term fibromyalgia syndrome for the condition. Other symptoms include debilitating fatigue, sleep disturbance, and joint stiffness. Some people also report difficulty with swallowing, bowel and bladder abnormalities, numbness and tingling, and cognitive dysfunction. Fibromyalgia is frequently associated with psychiatric conditions such as depression and anxiety and stress-related disorders such as posttraumatic stress disorder. Not all people with fibromyalgia experience all associated symptoms.

Although the diagnostic criteria for fibromyalgia presented by ACR (American College of Rheumatology) in 1990 was as listed in Table 1, as they were hard to apply on a clinical basis and similar patients who did not necessarily follow the same criteria, the ACR has announced a new version in 2010 (Table 2).

\section{EPIDEMIOLOGY, CAUSES AND PATHOPHYSIOLOGY OF FIBROMYALGIA}

Fibromyalgia is estimated to affect $2-8 \%$ of the population, with a female to male incidence ratio that is somewhere between $7: 1$ and $9: 1$. It is most commonly diagnosed in individ-

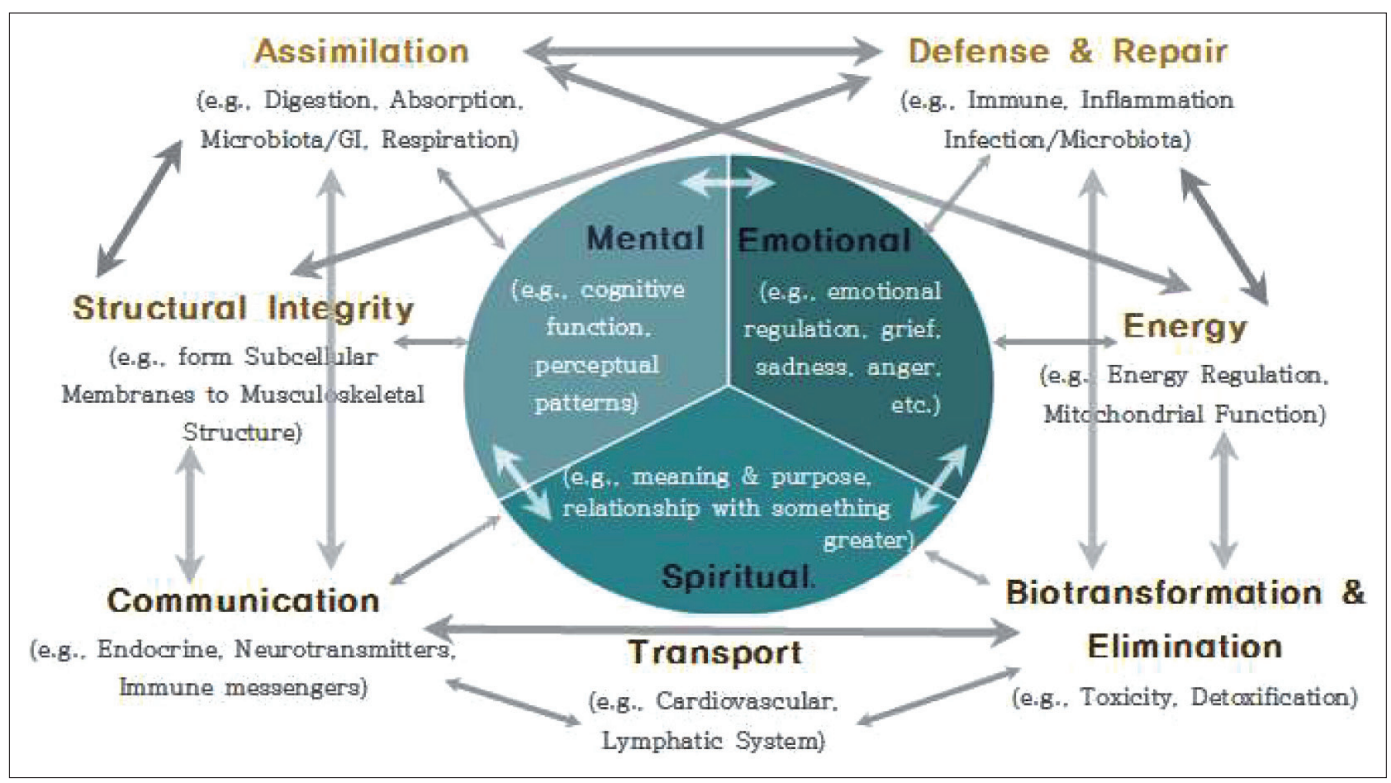

Fig. 3. The Functional medicine matrix. Functional medicine is an individualized approach to patient care that focuses on restoring balance to the dysfunctional systems by strengthening the fundamental physiologic processes that underlie them, and by adjusting the environmental inputs that nurture or impair them. Functional medicine looks at the triggers and mediators that lead to a particular clinical state. This takes into account the environmental factors that wash over a patient's particular genetic uniqueness that then can result in either vitality or dysfunction. The environmental effect can be as broad as the mind's influence on the psychoneuroendocrine system or as narrow as a conditionally essential nutrient in a metabolic pathway that has been affected by an environmental toxin. Functional medicine uses a matrix of core clinical imbalances to organize a structural framework around a patient's complex presentation. 


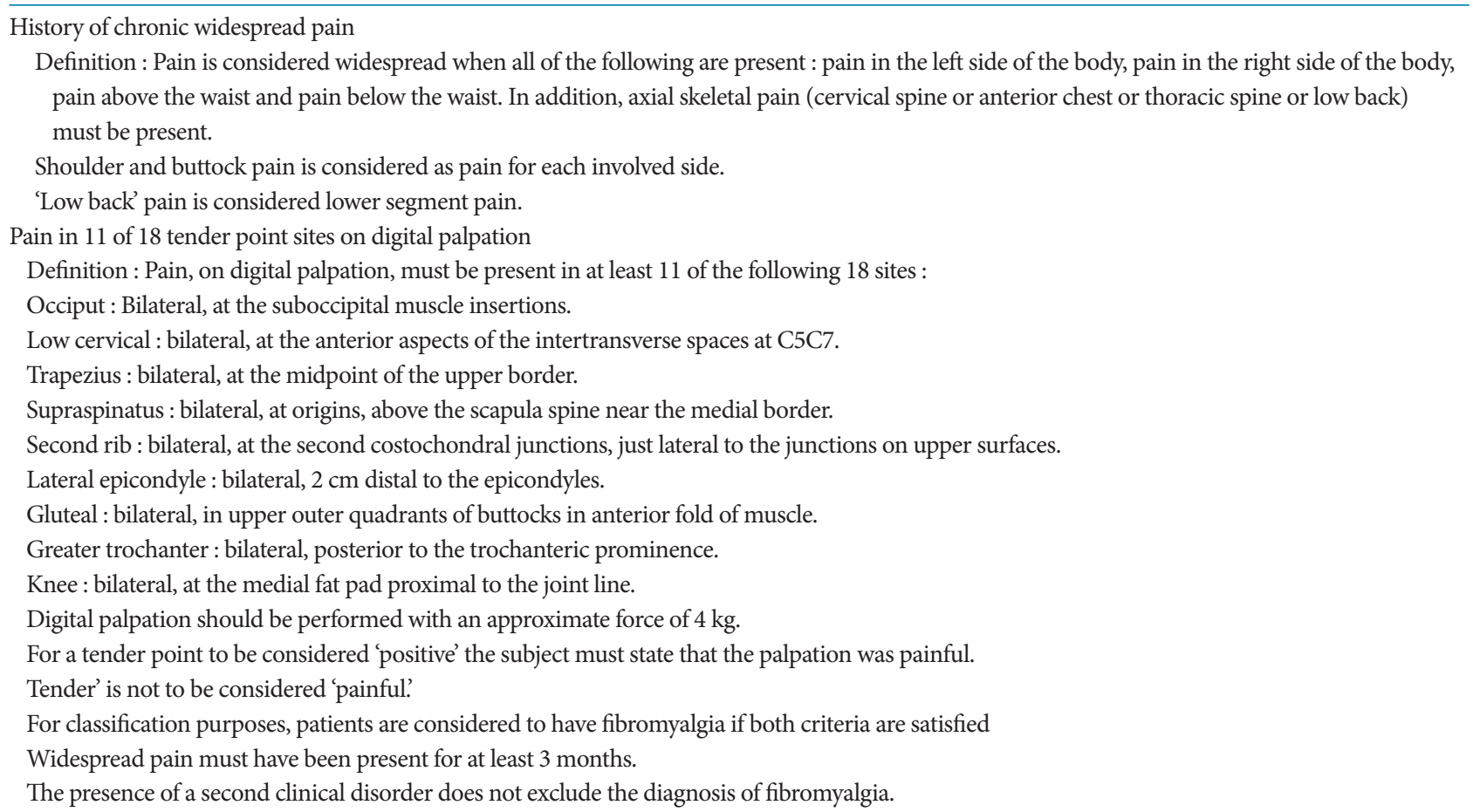

Table 2. The 2010 American College of Rheumatology Diagnostic Criteria

A patient satisfies diagnostic criteria for fibromyalgia if the following three conditions are met:

1. Widespread pain index (WPI $)^{\ddagger} 7$ and symptom severity (SS) scale score 5 or WPI 36 and SS scale score $e^{\ddagger} 9$.

2. Symptoms have been present at a similar level for at least 3 months.

3. The patient does not have a disorder that would otherwise explain the pain.

Ascertainment

1. Widespread Pain Index (WPI) : Note the number areas in which the patient has had pain over the last week. In how many areas has the patient

had pain? Score will be between 0 and 19.

Shoulder girdle, left; hip (buttock, trochanter), left; jaw, left; upper back

Upper arm, left; upper leg, left; chest; neck

Upper arm, right; upper leg, right; abdomen

Lower arm, left; lower leg, left

Lower arm, right; lower leg, right

2. SS scale score :

Fatigue, waking unrefreshed, cognitive symptoms

For the each of the three symptoms above, indicate the level of severity over the past week using the following scale :

$0=$ no problem

$1=$ slight or mild problems, generally mild or intermittent

$2=$ moderate, considerable problems, often present and/or at a moderate level

$3=$ severe : pervasive, continuous, life-disturbing problems

Considering somatic symptoms in general, indicate whether the patient has : $\dagger$

$0=$ no symptoms

$1=$ few symptoms

$2=$ a moderate number of symptoms

$3=$ a great deal of symptoms

uals between the ages of 20 and 50, though onset can occur in childhood. Fibromyalgia may not be diagnosed in up to 75 percent of affected people. The possible causes of fibromyalgia is psychological, genetic, neurobiological and environmental factors, But until now, the exact cause of fibromyalgia is unknown. The known pathophysiologic mechanisms are dopamine dysfunction, serotonin metabolism, poly-modal sensitivity, neuro- endocrine disruption, sympathetic hyperactivity and cerebrospinal fluid abnormalities. Nowadays, Fibromyalgia, a central nervous system disorder, is described as a "central sensitization syndrome" caused by neurobiological abnormalities which act to produce physiological pain and cognitive impairments as well as neuro-psychological symptomatology.

As it can be observed from Table 3, fibromyalgia is not just a 
disease with pain but one that involves various organs with autonomic dysfunctions.

This implies that a few medications including NSAIDs would just alleviate the patient's symptom partially, meaning not completely. When a human body is stressed, the limbic system, especially the anterior cingulate cortex (ACC), sends signals to hypothalamus so that the pituitary secretes ACTH in order to stimulate the adrenal cortex. As a result, the stress hormone, cortisol is secreted and the blood glucose increases. In order to decrease the blood glucose, insulin is secreted. Also, with the rise of gastric acid secretion, there could be dyspepsia, gastritis or peptic ulcer disease, and when the cortisol level is risen chronically, the immune system is suppressed, therefore debilitated when faced with infections (Fig. 4).

Therefore, to explain fibromyalgia accompanied with chronic pain, the psychoneuroendoimmune model is employed. Under this model, the central nervous system, neuroendocrine system and the immune system are known to make bidirectional interactions $^{31)}$.

The definition of dual tryptophan pathways has increased the understanding of the mind-body, body-mind dichotomy. The serotonergic pathway highlights the primary (endogenous) psychiatric disorders. The up-regulation of the kynurenine pathway by physical illnesses can cause neuropathic and immunological disorders associated with secondary neuropsychiatric symptoms. Tryptophan and nicotinamide deficiencies fall within the protein energy malnutrition (PEM) spectrum. They can arise if the kynurenine pathway is stressed by primary or secondary inflammatory conditions and the consequent imbalance of available catabolic/anabolic substrates may adversely influence convalescent phase efficiency. The replacement of depleted or reduced NAD+levels and other cofactors can perhaps improve the clinical management of these disorders. Chronic fatigue syndrome (CFS) and fibromyalgia (FM) appear to meet

Table 3. Common signs and symptoms of fibromyalgia

\begin{tabular}{ll}
\hline Central & Chronic headache, Sleep disorders, Dizziness, Cognitive impairment, Memory impairment, Anxiety, Depression \\
Muscular & Myofascial pain, Fatigue, Twitches, Tingling sensation \\
Urinary & Problems urinating, Dysuria, Frequency, Urgency \\
Eyes & Vision problems, Dry eye syndrome \\
Systemic & Pain, Weight gain, Cold symptoms, Multiple chemical sensitivity \\
Joint of jaw & Dysfunction, Malocclusion \\
Skin & Various complaints, Acne, Dryness, Itching \\
Chest region & Pain, Tightness \\
Stomach & Nausea, Irritable bowel syndrome, Constipation, Diarrhea \\
Joints & Morning stiffness, Multiple joint pain \\
\hline
\end{tabular}

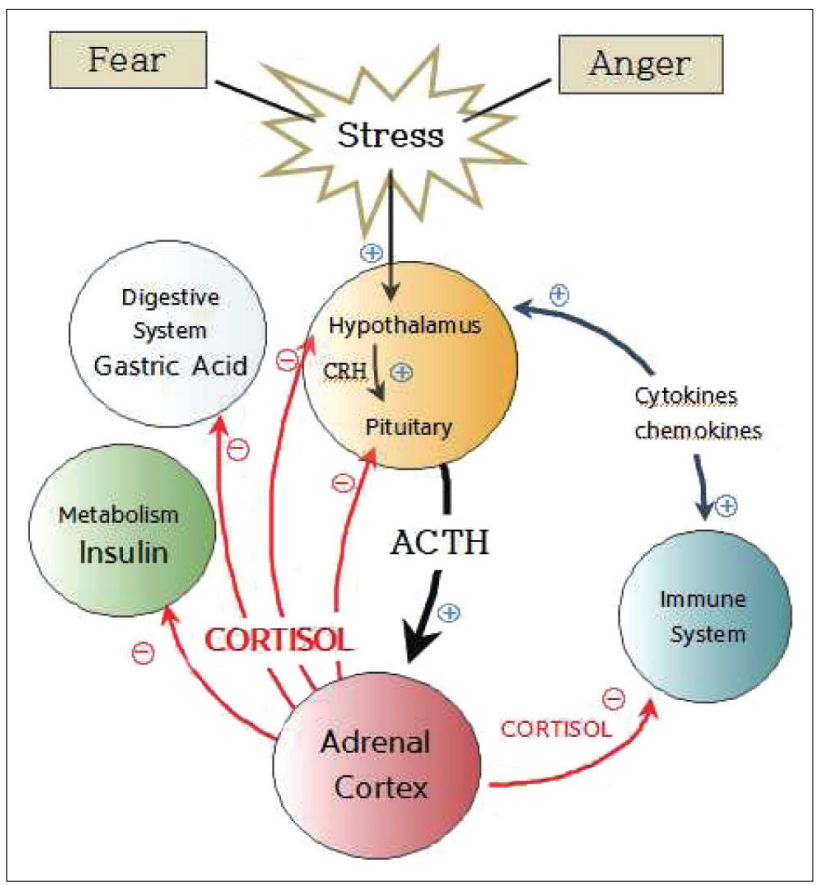

Fig. 4. Metabolic and system responses to stress ${ }^{19)}$. The hypothalamic, pituitary and adrenal glands respond in tandem to elicit multiple tissue responses when stress signals are received. When the brain perceives the removal of the threat, then the initiating neuronal signals subside and the system returns to basal levels of activity.

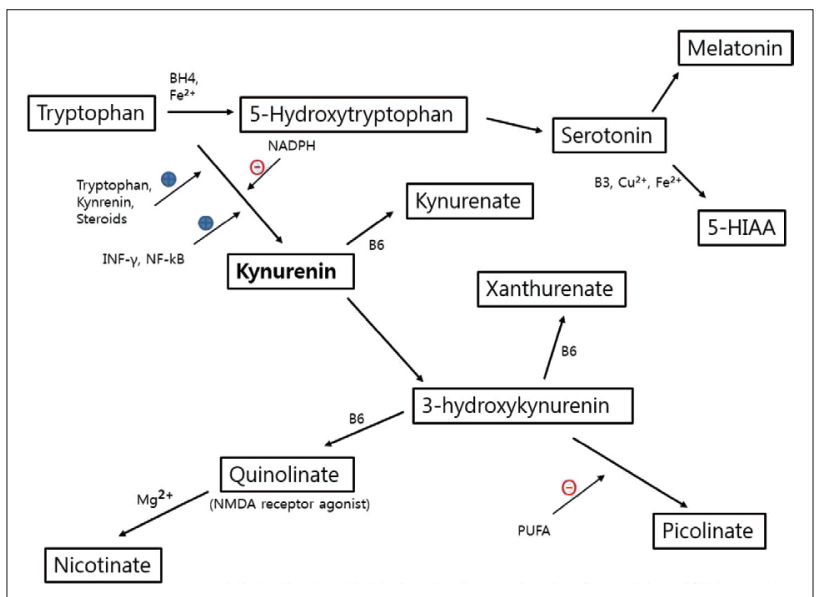

Fig. 5. Kynurenine pathway ${ }^{25}$. Entry of tryptophan into the kynurenin pathway is governed by tryptophan-2,3-dioxygenase that is subject to regulation by tryptophan, NADPH, kynurenin and steroid hormones. When all enzymes are properly formed and cofactor status is good, the pathway leads to either quinolinate or nicotinate, depending on tissue location. The critical point for vitamin $\mathrm{B}_{6}$ functional assessment is the pyridoxal-5-phosphate requiring kynurenase step. Cofactor insufficiency to sustain this enzyme activity causes diversion of 3-hydroxykynurenin to alternative pathways leading to production of xanthurenate and kynurenate. Omega-3 polyunsaturated fatty acids suppress transcription of ACMSD, decreasing picolinate production. NADPH : Nicotinamide Adenine Dinucleotide (reduced form), $\mathrm{B} 6$ : Vitamin $\mathrm{B} 6, \mathrm{Mg}^{2+}$ : ionized magnesium, PUFA : PolyUnsaturated Fatty Acid, B3: Vitamin B3, $\mathrm{Fe}^{2+}$ : ferrous ion. 
the criteria of a tryptophan-kynurenine pathway disorder with potential neuroimmunological sequelae (Fig. 5$)^{2}$.

Recent years added new information to our understanding of FM pathophysiology. Researches on genetics, biogenic amines, neurotransmitters, hypothalamic-pituitary-adrenal axis hormones, oxidative stress, and mechanisms of pain modulation, central sensitization, and autonomic functions in FM revealed various abnormalities indicating that multiple factors and mechanisms are involved in the pathogenesis of FM. Oxidative stress and nitric oxide may play an important role in FM pathophysiology, however it is still not clear whether oxidative stress abnormalities documented in FM are the cause or the effect. This should encourage further researches evaluating the potential role of oxidative stress and nitric oxide in the pathophysiology of FM and the efficacy of antioxidant treatments (omega-3 and -6 fatty acids, vitamins and others) in double blind and placebo controlled trials (Fig. 6) ${ }^{21)}$.

Research on different parameters in fibromyalgia (FM) indicates that multifactors are involved in its pathophysiology; such as genetic factors, substance $P$, serotonin, hypothalamic pituitary adrenal axis (HPA), muscles metabolic dysfunction, reactive oxygen species (ROS) and reactive nitrogen species (RNS). Oxidative stress has also been implicated in the pathophysiology of FM; therefore, supplementation with antioxidants may be important in modulation of the effects of ROS in patients with $\mathrm{FM}^{12)}$.
Recent another studies also have shown evidence demonstrating that oxidative stress may have a role in the pathophysiology of FM, however it is still not clear whether oxidative stress is the cause or the effect of the abnormalities documented in FM. Furthermore, it is also controversial the role of mitochondria in the pathophysiology of FM, however signs associated with mitochondrial dysfunction have been observed in FM. Mitochondria are also known to be strong producers of ROS, so have been related with the pathogenic mechanism of numerous diseases including FM. To this respect, it has been observed antioxidants therapies might be beneficial to improve the mitochondrial performance. Therefore, the dysfunction mitochondrial opens a great field of therapeutic research, for what it should start considering in the clinical medicine the boarding of the FM by means of therapy with antioxidant and drugs related to the mitochondrial biogenesis ${ }^{3)}$.

Chronic fatigue syndrome (CFS) and fibromyalgia (FM) are hypothesized that reactive oxygen species (ROS), caused by oxidative and nitrosative stress, by inhibiting mitochondrial function can be involved in muscle pain and central sensitization as typically seen in these patients. The current evidence regarding oxidative and nitrosative stress and mitochondrial dysfunction in CFS and FM is presented in relation to chronic widespread pain. Mitochondrial dysfunction has been shown in leukocytes of CFS patients and in muscle cells of FM patients, which could

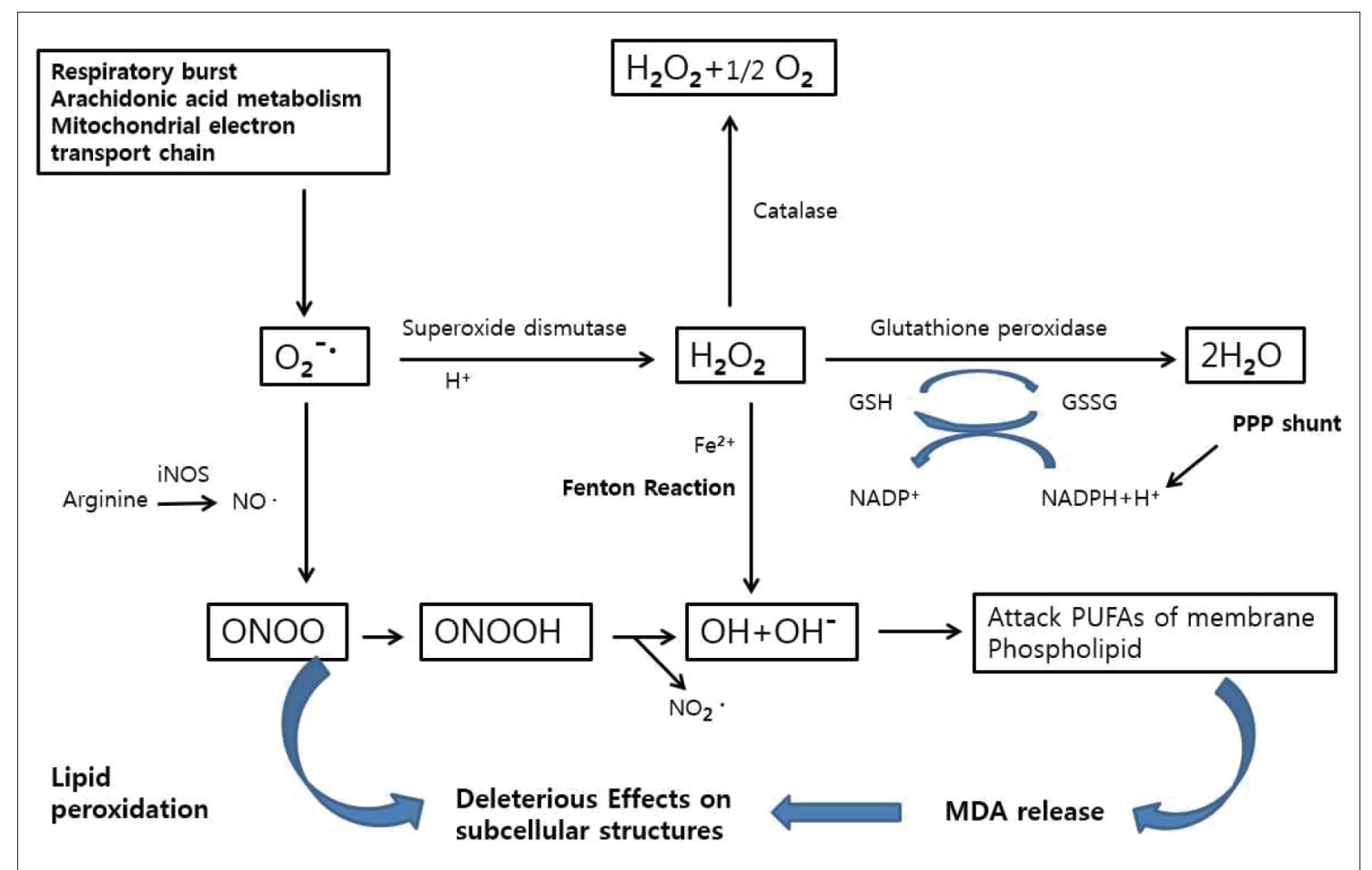

Fig. 6. Schematic view of free oxygen radical formation, enzymatic antioxidant and lipid peroxidation pathways ${ }^{21)} \cdot \mathrm{OH}^{\mathrm{O}}$ hydroxyl radical (the most potent free radical), $\mathrm{O}^{\bullet}-$-: superoxide anion radical, $\mathrm{O}^{*}:$ molecular oxygen, $\mathrm{OH}^{-}$: hydroxyl ion, ONOO: peroxynitrite, CAT: catalase, $\mathrm{Fe}^{++}$: ferrous iron, GSH: reduced glutathione, GSH-Red: glutathione reductase, GSSG: oxidized glutathione, $\mathrm{H}^{+}$: hydrogen ion, $\mathrm{H}_{2} \mathrm{O}$ : water, $\mathrm{H}_{2} \mathrm{O}_{2}$ : hydrogen peroxide, MDA: malondialdehyde (thiobarbituric acid reactive substance, an end-product of lipid peroxidation of membrane phospholipids), $\mathrm{NO}_{2}$ : nitrite, $\mathrm{NADP}^{+}$: oxidized nicotinamide adenine dinucleotide phosphate, $\mathrm{NADPH}+\mathrm{H}^{+}$: reduced nicotinamide adenine dinucleotide phosphate, PUFAs: polyunsaturated fatty acids, SOD: superoxide dismutase, tNOS: total nitric oxide synthases (neuronal NOS, endothelial NOS, and inducible NOS), NO ' : nitric oxide radical, XO: xanthine oxidase, GSH: reduced glutathione, GSSH: oxidized glutathione, PPP shunt: Pentos Phosphate Shunt, NADPH: Nicotinamide Adenine Dinucleotide Phosphate (reduced form). 
explain the muscle pain. Additionally, if mitochondrial dysfunction is also present in central neural cells, this could result in lowered ATP pools in neural cells, leading to generalized hypersensitivity and chronic widespread pain. Increased ROS in CFS and FM, resulting in impaired mitochondrial function and reduced ATP in muscle and neural cells, might lead to chronic widespread pain in these patients. Therefore, targeting increased ROS by antioxidants and targeting the mitochondrial biogenesis could offer a solution for the chronic pain in these patients. The role of exercise therapy in restoring mitochondrial dysfunction remains to be explored, and provides important avenues for future research in this area ${ }^{19)}$.

Putting together recent literature on fibromyalgia, it can be said that it arises from dysregulation of psychoneuroendoimmune system and mitochondriopathy causing from oxidative stress. There are two ways to measure the psychoneuroendoimmune system; salivary free cortisol and DHEA (dehydroepiandrosterone). The level of cortisol collected from saliva can only be measured in biologically active free form not bind with proteins $^{1,18,28)}$.

In the early stage of stress, DHEA and cortisol levels increase at the same time, but as stress lingers, DHEA first starts declin-

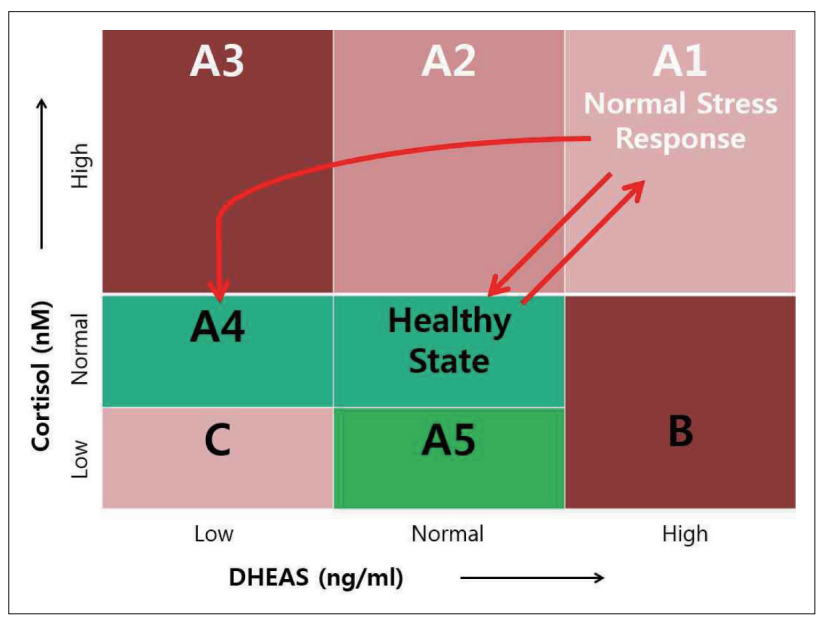

Fig. 7. Stress responses of cortisol and DHEA ${ }^{27)}$. The values that are plotted are the average of noon and 5 p.m. levels for salivary cortisol and DHEA. The quadrant represent normal and abnormal patterns, with $\mathrm{A} 1$ to $\mathrm{A} 4$ representing progressive failure of the normal ACTH response.

Table 4. $5 \mathrm{R}$ for gastrointestinal function support program and optimal gut health

\begin{tabular}{ll}
\hline Remove & $\begin{array}{l}\text { Pathogenic/Xenobiotics, Allergens } \\
\text { Examples; Gluten, Dairy, Corn, Sugar free diet }\end{array}$ \\
Replace & $\begin{array}{l}\text { Digestive enzyme and factors (example; betaine HCl) } \\
\text { Reinoculate }\end{array}$ \\
& $\begin{array}{l}\text { Probiotics; bifidobacteria, lactobacilli } \\
\text { Prebiotics: fructooligosaccharide(FOS), inulin }\end{array}$ \\
Repair & $\begin{array}{l}\text { Low irritant diet } \\
\text { Nutrient to support growth and repair }\end{array}$ \\
& $\begin{array}{l}\text { Examples: Glutamine/Licorice/Flavonoids/Slippery Elm } \\
\text { Rebalance }\end{array}$ \\
& $\begin{array}{l}\text { Pay attention to life style choices - sleep, exercise and } \\
\text { stress can all affect the GI tract (healthy eating, regular } \\
\text { exercise and stress management) }\end{array}$ \\
\hline
\end{tabular}

ing and finally the cortisol also decreases. If this is the case for a fibromyalgia-diagnosed patient, she/he would be accompanied with chronic fatigue syndrome and thus in a situation difficult to treat with (Fig. 7).

For want of space, the urinary organic acid test is not explained in detail, but through these tests, we can measure metabolites in course of metabolic process such as oxidative stress and TCA cycle, thereby indirectly measuring the deficiency of cofactor nutrients.

From a functional medicinal approach, the most important thing when it comes to treating patients is the function of gastrointestinal tract, in brief, the process of digestion-absorption-excretion should work well. There are 5Rs used as the principle for this approach (Table 4).

According to recent studies, the main cause for fibromyalgia is known to be increase of oxidative stress and decrease of mitochondrial energy production, so one should concentrate on normalizing these problems. First, sleep hygiene should be improved to help sleep well. Herbs or melatonin are put to use to help relaxation and good sleep, respectively. And the melatonin also have the antioxidant function, which mechanisms are direct free radical scavenging, stimulation of antioxidative enzyme and increasing the efficiency of mitochondrial oxidative phosphorylation, reducing electron leakage (thereby lowering free radical generation) and augmenting the efficiency of other antioxidant $^{24}$. Second, we need hormonal support, which optimizes the state of adrenal, thyroid, testosterone and estrogen. Also, nutritional support is needed to eliminate toxins and infections from hidden invaders and to maximize body healing. Patients with fibromyalgia is generally accelerated toward sympathetic nerve system rather than parasympathetic nerve system, thus relaxation treatments are in great need. Therefore, the concept of mind-body therapy is frequently employed, among which meditation, EFT (Emotional Freedom Technique), NLP (Neuro-Linguistic Programming) and deep breathing are examples for the relaxation treatment.

In conventional medicine, many chronic diseases including fibromyalgia are treated preferentially firsthand with drugs that instantly suppress the symptoms. However, in functional medicine, the fundamental cause of the disease, such as fibromyalgia, is traced to solve the pain, which is a medicine of new paradigm. It is considered that in order for the functional medicine to get settled as 21 st century medicine, plenty of researches and institutional supports are desperate.

\section{REFERENCES}

1. Aardal-Eriksson E, Karlberg BE, Holm AC : Salivary cortisol--an alternative to serum cortisol determinations in dynamic function tests. Clin Chem Lab Med 36 : 215-222, 1998

2. Blankfield A : Kynurenine pathway pathologies : do nicotinamide and other pathway co-factors have a therapeutic role in reduction of symptom severity, including chronic fatigue syndrome (CFS) and fibromyalgia (FM). Int J Tryptophan Res 6 (Suppl 1) : 39-45, 2013

3. Cordero MD, de Miguel M, Moreno-Fernández AM : Mitochondrial dys- 
function in fibromyalgia and its implication in the pathogenesis of disease. Med Clin (Barc) 136 : 252-256, 2011

4. Drug deaths now outnumber traffic fetalities in U.S. data (L.A. Times, September 17, 2011)

5. Egger G, Dixon J : Should obesity be the main game? Or do we need an environmental makeover to combat the inflammatory and chronic disease epidemics? Obes Rev $10: 237-249,2009$

6. Ely JW, Osheroff JA, Gorman PN, Ebell MH, Chambliss M, Pifer EA, et al. : A taxonomy of generic clinical questions : classification study. BMJ 321 : 429-432, 2000

7. Gareau MG, Silva MA, Perdue MH : Pathophysiological mechanisms of stress-induced intestinal damage. Curr Mol Med 8 : 274-281, 2008

8. Gislason GH : Duration of treatment with nonsteroidal anti-inflammatory drugs and impact on risk of death and recurrent myocardial infarction in patients with prior myocardial infarction : a nationwide cohort study. Circulation 123 : 2226-2235, 2011

9. Gislason GH : Risk of death or reinfarction associated with the use of selective cyclooxygenase-2 inhibitors and nonselective nonsteroidal antiinflammatory drugs after acute myocardial infarction. Circulation 113 : 2906-2913, 2006

10. Gislason GH, Olsen AM : Long-term cardiovascular risk of nonsteroidal anti-inflammatory drug use according to time passed after first-time myocardial infarction : a nationwide cohort study. Circulation 126 : 19551963,2012

11. Holman $\mathrm{H}$ : Chronic disease-The need for a new clinical education. JAMA 292 : 1057-1059, 2004

12. Iqbal R, Mughal MS, Arshad N, Arshad M : Pathophysiology and antioxidant status of patients with fibromyalgia. Rheumatol Int 31 : 149-152, 2011

13. Jones DS : Changing the evidence model in Jones DS (ed) : Textbook of Functional Medicine. Gig Harbor, WA : The Institute for Functional Medicine, 2005

14. Johns MME, Brigham KL: Transforming health care through prospective medicine : The first step. Acad Med 83 : 706, 2008

15. Kivimäki M, Virtanen $M$, Elovainio $M$, Kouvonen A, Vänänen A, Vahtera J. Work stress in the etiology of coronary heart disease-a meta-analysis. Scand J Work Environ Health 32 : 431-442, 2006

16. Kumanyika SK, Obarzanek E, Stettler N, Bell R, Field AE, Rortmann SP, et al. : Population based prevention of obesity. The need for comprehensive promotion of healthful eating, physical activity, and energy balance. Circulation $118: 428-464,2008$

17. Logan JG, Barksdale DJ : Allostasis and allostatic load : expanding the discourse on stress and cardiovascular disease. J Clin Nurs 17 : 201-208, 2008

18. Maidana $\mathrm{P}$, Bruno OD, Mesch V : A critical analysis of cortisol measurements : an update. Medicina (B Aires) $73: 579-584,2013$

19. Meeus M, Nijs J, Hermans L, Goubert D, Calders P : The role of mitochondrial dysfunctions due to oxidative and nitrosative stress in the chronic pain or chronic fatigue syndromes and fibromyalgia patients : peripheral and central mechanisms as therapeutic targets? Expert Opin Ther Targets $17: 1081-1089,2013$

20. O’Toole TE, Conklin DJ, Bhatnagar A : Environmental risk factors for heart disease. Rev Environ Health 23 : 167-202, 2008

21. Ozgocmen S, Ozyurt H, Sogut S, Akyol O : Current concepts in the pathophysiology of fibromyalgia : the potential role of oxidative stress and nitric oxide. Rheumatol Int 26 : 585-597, 2006

22. Pan SY, DesMeules M : Energy intake, physical activity, energy balance, and cancer : epidemiologic evidence. Methods Mol Biol 472 : 191-215, 2009

23. Ramos RG, Olden $\mathrm{K}$ : Gene-environment interactions in the development of complex disease phenotypes. Int J Environ Res Public Health 5 : 4-11, 2008

24. Reiter RJ, Tan DX, Mayo JC, Sainz RM, Leon J, Czarnocki Z : Melatonin as an antioxidant : biochemical mechanisms and pathophysiological implications in humans. Acta Biochim Pol 50 : 1129-1146, 2003

25. Richard S, Lord J, Bralley A : Laboratory evaluations for integrative and functional medicine, 2nd edition, p356

26. Richard S, Lord J, Bralley A : Laboratory evaluations for integrative and functional medicine, 2nd edition, p549.

27. Richard S, Lord J, Bralley A : Laboratory evaluations for integrative and functional medicine, 2nd edition, p561

28. Törnhage CJ : Salivary cortisol for assessment of hypothalamic-pituitaryadrenal axis function. Neuroimmunomodulation 16 : 284-289, 2009

29. US data show sharply rising drug-induced death rates, NCHS Data Brief No. 81 December 2011. Injury Prevention 13: 30-132, 2007

30. Voelker R : US health care system earns poor marks. JAMA $300: 2843-$ 2844, 2008

31. Yocum DE, Castro WL, Cornett M : Exercise, education, and behavioral modification as alternative therapy for pain and stress in rheumatic disease. Rheum Dis Clin North Am 26 : 145-159, 2000

32. 21st century medicine: A New Model for Medical Education and Practice, xii 2009 IFM, David S. Jones, MD 